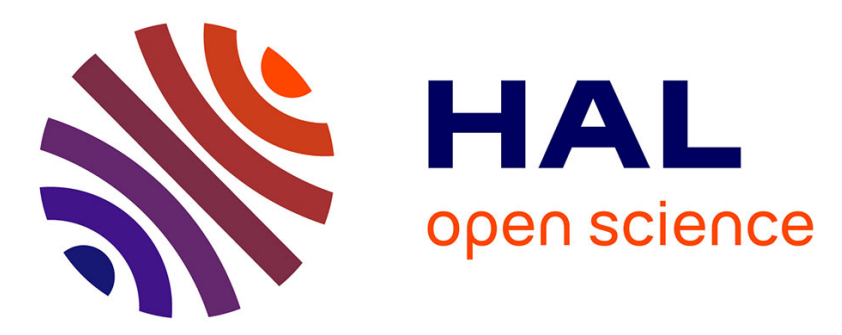

\title{
Lowering of ground state induced by core-shell structure in strontium titanate
}

Jean-Michel Kiat, Bernard Hehlen, Mickaël Anoufa, Christine Bogicevic, A M Curfs, Bernard Boyer, A M Al-Sabbagh, F M Porcher, A M Al-Zein

\section{- To cite this version:}

Jean-Michel Kiat, Bernard Hehlen, Mickaël Anoufa, Christine Bogicevic, A M Curfs, et al.. Lowering of ground state induced by core-shell structure in strontium titanate. Physical Review B, 2016, 93, pp.144117 - 144117. 10.1103/PhysRevB.93.144117 . hal-01384969

\section{HAL Id: hal-01384969 \\ https://hal.science/hal-01384969}

Submitted on 20 Oct 2016

HAL is a multi-disciplinary open access archive for the deposit and dissemination of scientific research documents, whether they are published or not. The documents may come from teaching and research institutions in France or abroad, or from public or private research centers.
L'archive ouverte pluridisciplinaire HAL, est destinée au dépôt et à la diffusion de documents scientifiques de niveau recherche, publiés ou non, émanant des établissements d'enseignement et de recherche français ou étrangers, des laboratoires publics ou privés. 


\title{
Lowering of ground state induced by core-shell structure in strontium titanate
}

\author{
J. M. Kiat,,${ }^{1,2,{ }^{*}}$ B. Hehlen, ${ }^{3}$ M. Anoufa, ${ }^{1}$ C. Bogicevic, ${ }^{1}$ C. Curfs ${ }^{4}$ B. Boyer, ${ }^{5}$ M. Al-Sabbagh, ${ }^{3}$ F. Porcher, ${ }^{2}$ and A. Al-Zein ${ }^{4}$ \\ ${ }^{1}$ Laboratoire Structures, Propriétés et Modélisation des Solides, Ecole Centrale Paris, CNRS-UMR 8580, Grande Voie des Vignes, \\ 92295 Chatenay-Malabry Cedex, France \\ ${ }^{2}$ Laboratoire Léon Brillouin, CE Saclay CNRS-UMR 12, 91991 Gif-Sur-Yvette Cedex, France \\ ${ }^{3}$ Laboratoire Charles Coulomb, CNRS-UMR 5221, Université Montpellier II-CC 069 Place E. Bataillon, 34095 Montpellier, France \\ ${ }^{4}$ European Synchrotron Radiation Facility, 71 Avenue des Martyrs, 38000 Grenoble Cedex, France \\ ${ }^{5}$ Géoscience Montpellier, CNRS-UMR 5342, Université Montpellier, Montpellier, France
}

(Received 6 January 2016; revised manuscript received 30 March 2016; published 19 April 2016)

\begin{abstract}
A new ground state of textbook compound strontium titanate $\left(\mathrm{SrTiO}_{3}\right)$ is obtained by inducing a specific core-shell structure of the particles. Using a combination of high energy synchrotron and neutron diffraction, we demonstrate a lowering of the ferroelastic ground state towards a new antiferrodistortive phase, accompanied with strong shifts of the critical temperature. This new phase is discussed within the Landau theory and compared with the situation in thin films and during pressure experiments. The crucial competition between particle shape anisotropy, surface tension, and shear strain is analyzed. Inducing a specific core-shell structure is therefore an easy way to tailor structural properties and to stabilize new phases that cannot exist in bulk material, just like film deposition on a substrate.
\end{abstract}

DOI: 10.1103/PhysRevB.93.144117

\section{INTRODUCTION}

Most technological applications in metallurgy, civil engineering, and microelectronic require mastering synthesis and shaping of basic materials in the form of powders. In all these fields, an increasing need for smaller sizes, better purity, and better homogeneity is required, and many experimental and theoretical efforts are devoted to understand the consequences of downscaling. Focusing on the impressive successes of microelectronic associated with the ability of chipset manufacturers to integrate more and more components (as described by Moore's law), semiconductors and magnetic and dielectric materials have revealed many new fascinating features at smaller sizes. Indeed, below some microns, new properties can emerge, motivating continuous efforts for going to smaller and smaller sizes.

From a physical point of view, as size is getting smaller, competition between surface and volumic energy is increasing, but this sole effect does not accurately describe many experimental situations in which the increase of internal strain is also at play. This is, of course, peculiarly important in the case of thin films in which deposition on substrate with different lattice parameters obviously induces misfit strain that can be locally extremely high. These effects have been studied in ferroelectric oxide films used in multilayer capacitors, actuators, memories, etc., and many new structures and complex domain patterns not possible in bulk materials have been evidenced. However, structural changes induced by strain can be also encountered in particles, as small grain sizes do not allow easy relaxation of stress, such as the one induced during synthesis or by creation of domains. In this case, lowering of the ground state (with rotation of polarization and vanishing of dielectric relaxation) has been reported and explained on the basis of size and strain effects [1]. However, in most of the textbook ferroelectric and related compounds, more sophisticated models are needed

\footnotetext{
*jean-michel.kiat@ecp.fr
}

to account for effects, such as size dependence of dielectric properties: In this case, size/strain competition is not sufficient, and the core-shell structure (CSS) of grains must be taken into account. This situation describes the structuration of particles, where the core behaves like the bulk material, whereas the shell, with a typical thickness of some nanometers and a low-permittivity (typically $\epsilon \approx 100$ ), is the part of the particle close to the surface, where all kinds of defects are at play.

This is well known in the case of thin films in which a "dead layer" often appears close to the surface, but before these observations in films, a CSS structure was reported [2] in the prototype ferroelectrics $\mathrm{BaTiO}_{3}$ (BTO) and $\mathrm{PbTiO}_{3}$ (PTO), quantum paraelectric $\mathrm{SrTiO}_{3}$ (STO), and more recently in lead-based relaxor and morphotropic compounds [3]. In some cases, i.e., in $\mathrm{STO}$ or in relaxor $\mathrm{PbMg}_{1 / 3} \mathrm{Nb}_{2 / 3} \mathrm{O}_{3}$ (PMN), a double shell has been assumed, where the closest part to the surface has even smaller permittivity $(\epsilon \approx 5)$. The precise microscopic structure of this shell (or dead layer) is yet unclear in many compounds, as it strongly depends on the synthesis route, the sintering process, the presence of impurity ions, or vacancies. In the case of the prototype PMN relaxor, a shell of $\mathrm{PbO}$ coating the inner surface has been observed [4], which successfully explains the size dependence of permittivity. In many ferroelectrics, it is also possible to induce artificial CSS either by doping with ions that diffuse weakly inside the particles and remain mainly close to the surface of the grain or by coating the grains with another compound with much lower permittivity, typically silica $\mathrm{SiO}_{2}$ or alumina $\mathrm{Al}_{2} \mathrm{O}_{3}$ (e.g., Ref. [5]). Also, during the sintering of powders to form ceramics, grain boundaries very often become uncontrolled defective structures different from the bulk. Therefore, we can refer to "natural" or "artificial" (i.e., induced) CSS, depending on the context and the synthesis. In all cases, the existence of this CSS can be used to tailor physical properties: This is classically done in microelectronic companies that prepare the so-called $\mathrm{X} 7 \mathrm{R}$ capacitors whose performances have a very "flat" thermal dependence. Others examples are recent calculation of optimal CSS structures to maximize energy 
storage [6] and electrocaloric effect [7] in most classical ferroelectric ceramics. Structural changes induced by CSS have already been reported in metallic core or shell $\mathrm{Fe} / \mathrm{Cu}$ and $\mathrm{Fe} / \mathrm{Au}$ nanoparticles prepared by deposition from the gas phase (see Ref. [8] and reference herein). We provide here experimental proof in an insulating compound of such changes and a phenomenological framework of interpretation.

In this paper, we pay attention to $\mathrm{SrTiO}_{3}$ (STO), which is a quantum paraelectric (or incipient ferroelectric) compound at very low temperatures, i.e., below $30 \mathrm{~K}$. The physical picture is that quantum fluctuations (zero-point motion) and eventually the ferroelastic antiferrodistortive (AFD) transition [9] that happens below $105 \mathrm{~K}$ suppress the latent ferroelectricity; this compound is therefore "at the edge" of ferroelectricity, for the following reasons. First, it displays very high values of permittivity saturating at very low temperature. Moreover, changes in chemical composition (i.e., addition of a cation, such as $\mathrm{Ba}, \mathrm{Ca}$, or $\mathrm{O} 16 / \mathrm{O} 18$ replacement), application of pressure or electric field, and deposition in the form of epitaxial thin films, can stabilize a short-range or long-range ferroelectric polarization. Finally, nanoclusters of polarization have been evidenced [10], very reminiscent of the polar nanoregions of relaxors. Thus, there are many similarities between ferroelectrics, relaxors, and quantum paraelectric compounds, and a progressive transformation between these three types of properties has been seen in $\mathrm{Li}$-doped $\mathrm{KTiO}_{3}$, depending on the Li concentration [11]. Natural CSS induced by oxygen vacancies at the surface has been assumed in STO by Petzelt [2] to explain the size dependence of dielectric permittivity, as well as many other observations such as infrared and Raman scattering. In this paper, we presents structural and modeling results on artificial $\mathrm{SrTiO}_{3} / \mathrm{ZrO}_{2}$ core-shell system: We want to show that by controlling the nature of the shell, as in $\mathrm{STO} / \mathrm{ZrO} 2$ core-shell system, changes in the structural properties can be obtained in STO particles.

\section{EXPERIMENTAL}

We have prepared an artificial CSS in STO by synthesizing micrometric powders using a conventional route whose size was afterward reduced by a ball milling technique in a $\mathrm{ZrO}_{2}$ bowl down to $\sim 15 \mathrm{~nm}$. These nanopowders were annealed at different temperatures to obtain ceramics with increasing grain sizes. Ceramics and grinded powders, with sizes ranging from $15 \mathrm{~nm}$ to $\sim 1 \mu \mathrm{m}$ and typical distribution checked by transmission electron microscope (TEM), scanning electron microscopy, and by $x$-ray of $\cong 10 \%$ of the mean grain size, were obtained. Special attention was paid to avoid undesirable stress by performing further heat treatments at intermediate temperatures. For comparison, we also prepared STO particles in the same sizes range using a tungsten carbide (WC) bowl to obtain "natural" CSS STO, i.e., with oxygen vacancies at the surface.

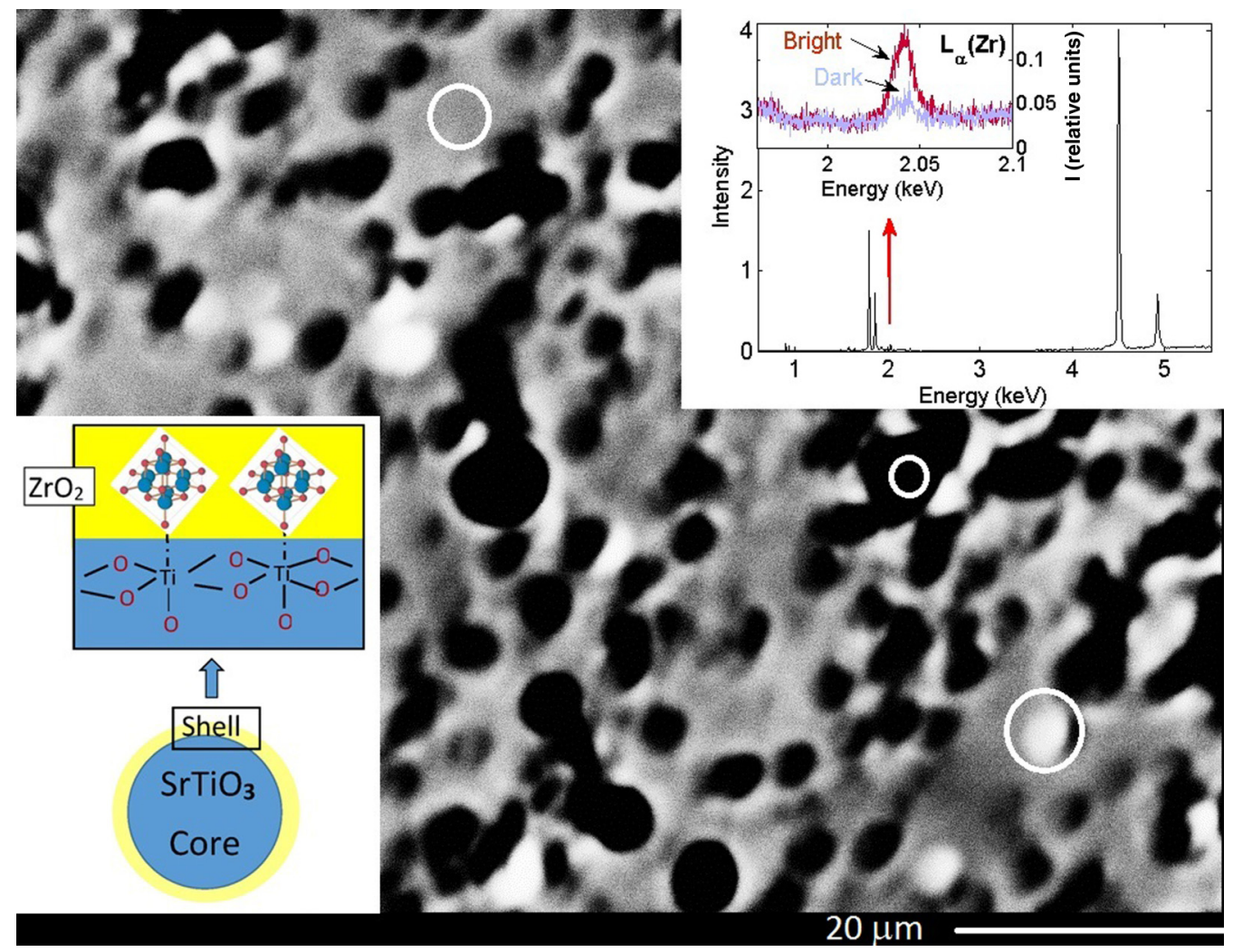

FIG. 1. The EPMA of STO ceramic: The x-ray mapping micrograph of the surface of micrometric artificial CSS STO ceramic obtained by EPMA. The grey contrast qualitatively reproduces the distribution of $\mathrm{Zr}$ at the surface. A typical x-ray spectrum is shown in the top right inset with a zoom on the weak $L_{\alpha}$ band of $\mathrm{Zr}$ in the dark and bright regions (white circles in the surface image). The black regions correspond to porosity at the surface. Left bottom inset is a sketch of the core-shell induced by $\mathrm{ZrO}_{2}$ grafting at the surface of the STO particles due to oxygen vacancies on the surface of the core. 
Electron probe microanalysis (EPMA) was performed using wavelength dispersive spectrometers (WDS) on a CAMECA SX100 at the University of Montpellier, France, to record the elemental spectrum. One of the spectrometers equipped with a thallium acid phthalate crystal permitted us to record the wavelength between 0.54 and $2.11 \mathrm{~nm}$, corresponding to a $2.31-0.59 \mathrm{keV}$ energy bandwidth, while another spectrometer with a large pentaerythritol crystal explored the $0.18-0.72 \mathrm{~nm}(6.81-1.73 \mathrm{keV})$ band. Incident electron beam intensity was $40 \mathrm{nA}$ with an energy of $20 \mathrm{keV}$.

The x-ray diffraction experiments were performed on ID22 and ID31 at the European Synchrotron Radiation Facility (ESRF), Grenoble, France. A channel cut Si (111) monochromator was used to provide an incident beam with wavelength $\lambda=0.40 \AA$ A . Diffraction patterns in the angular scattering range 158 between 5 and $90^{\circ}$ were recorded between 10 and $300 \mathrm{~K}$ using a liquid-helium-cooled cryostat. Neutron diffraction measurements were made with a two-axis high resolution 3T2 diffractometer at Laboratoire Léon Brillouin (LLB), Saclay, France. The Ge (335) curved monochromator was used to provide an incident beam with a wavelength $\lambda=1.226 \AA$. Diffraction patterns in the angular scattering range between 4 and $121^{\circ}$ were recorded between 2 and $300 \mathrm{~K}$ using a liquid-helium-cooled cryostat.

\section{RESULTS}

\section{A. Samples morphology}

The TEM and EPMA of artificial CSS STO ceramics revealed that during the ball milling process, $\mathrm{ZrO}_{2}$ particles were pulled off from the bowl and spread by subsequent heating to form a coating all over the STO grains (Fig. 1). High energy $\mathrm{x}$-ray synchrotron experiments also evidenced weak and diffuse $\mathrm{ZrO}_{2}$ diffraction peaks in addition to the STO Bragg peaks, with a corresponding volumic amount similar to the one measured by EPMA (between 0.12 and $0.22 \%$, depending on the samples). The physical origin of the grafting of $\mathrm{ZrO}_{2}$ around STO particles is induced by the oxygen nonstoichiometry of STO surfaces and grain boundaries just like in the natural CSS particles (Fig. 1). This nonstoichiometry has the consequence to create incomplete oxygen octahedra that act as effective oxygen vacancies and lead to pentagon environments formed on both $\mathrm{SrO}$ and $\mathrm{TiO}_{2}$ sublattices with a positive boundary charge. Therefore, oxygen atoms from $\mathrm{ZrO}_{2}$ fill the vacancies at the surface. For the "natural" CSS STO ceramics, no structural evidences (TEM, x-ray, etc.) of any "artificial" coating could be obtained in these samples, which is coherent with the picture that WC particles cannot graft on an oxygen-defective surface. The CSS structure of these samples that is required to explain dielectric properties at smaller sizes is therefore "natural" in the sense that it is consisting of oxygen-defective STO surface layers with low permittivity, which is more or less unavoidable.

\section{B. The x-ray and neutron diffraction measurements}

During the synchrotron diffraction experiments on ID22 and ID31 at the ESRF, full diffraction patterns with more than 330 peaks (in pseudocubic indexation), ranging between $\sin \theta / \lambda 0.1$ and 1.8 were recorded at $\lambda=0.40 \AA$ between $10 \mathrm{~K}$ (Fig. 2) and $300 \mathrm{~K}$ in both types of CSS samples, with
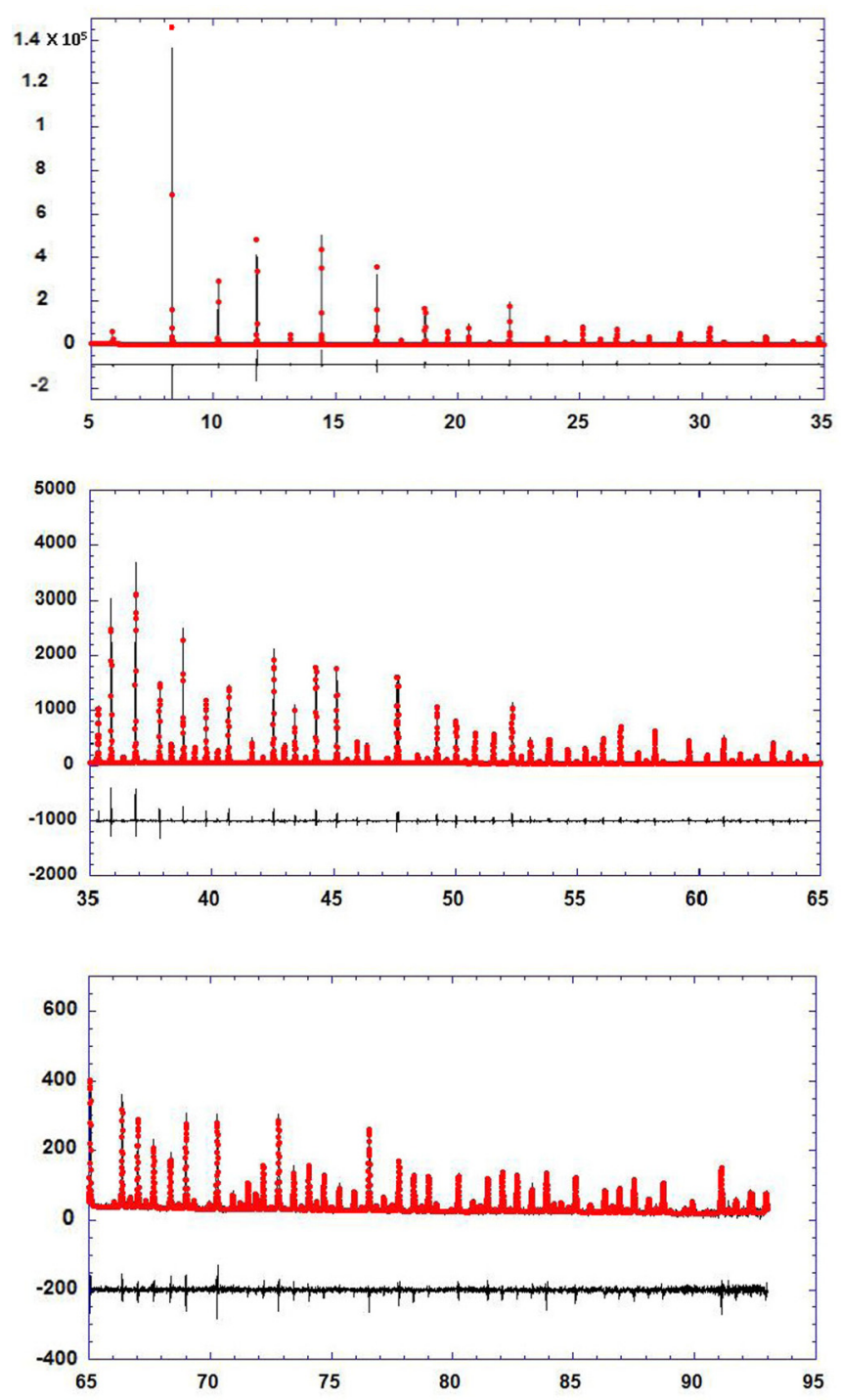

FIG. 2. The x-ray synchrotron full pattern at $10 \mathrm{~K}$ of micrometric artificial CSS STO, with calculated lines and difference using the final Rietveld model (see below).

different grain sizes. Complete sets of size and temperature structural evolutions were extracted from Rietveld analysis using Jana2006 [12]. In all samples, we have observed at $10 \mathrm{~K}$ splitting of the cubic $(H O 0)$ and $(H H O)$ peaks (Fig. 3), indicative of a distortion from the cubic phase, and small superstructure peaks (more clearly observed in neutron diffraction, see below) associated to the AFD motion of oxygen atoms during $\phi$ tilting of $\mathrm{TiO}_{6}$ octahedra at the ferroelastic transition. However for artificial CSS samples, an additional splitting of the (HHH) peaks (Figs. 3 and 4), which should not exist with the classical tetragonal distortion (space group $I 4 / \mathrm{mcm}$ ), see Table I, was detected at low temperature. This surprising splitting was observed for samples with micronic grain sizes and could be followed for samples with lower sizes until the broadening of peaks due to size effect becomes too strong and masks this splitting (i.e., down to $\approx 400 \mathrm{~nm}$ ). Monitoring the temperature dependence of both splitting (Fig. 4) and $\phi$ tilting (using neutron diffraction, see below, 


\section{Core-Shell $\mathrm{SrTiO}_{3}$ with $\mathrm{ZrO}_{2} \quad \mathrm{~T}=10 \mathrm{~K} \quad \mathrm{X}$-ray $\lambda=0.400 \AA$}
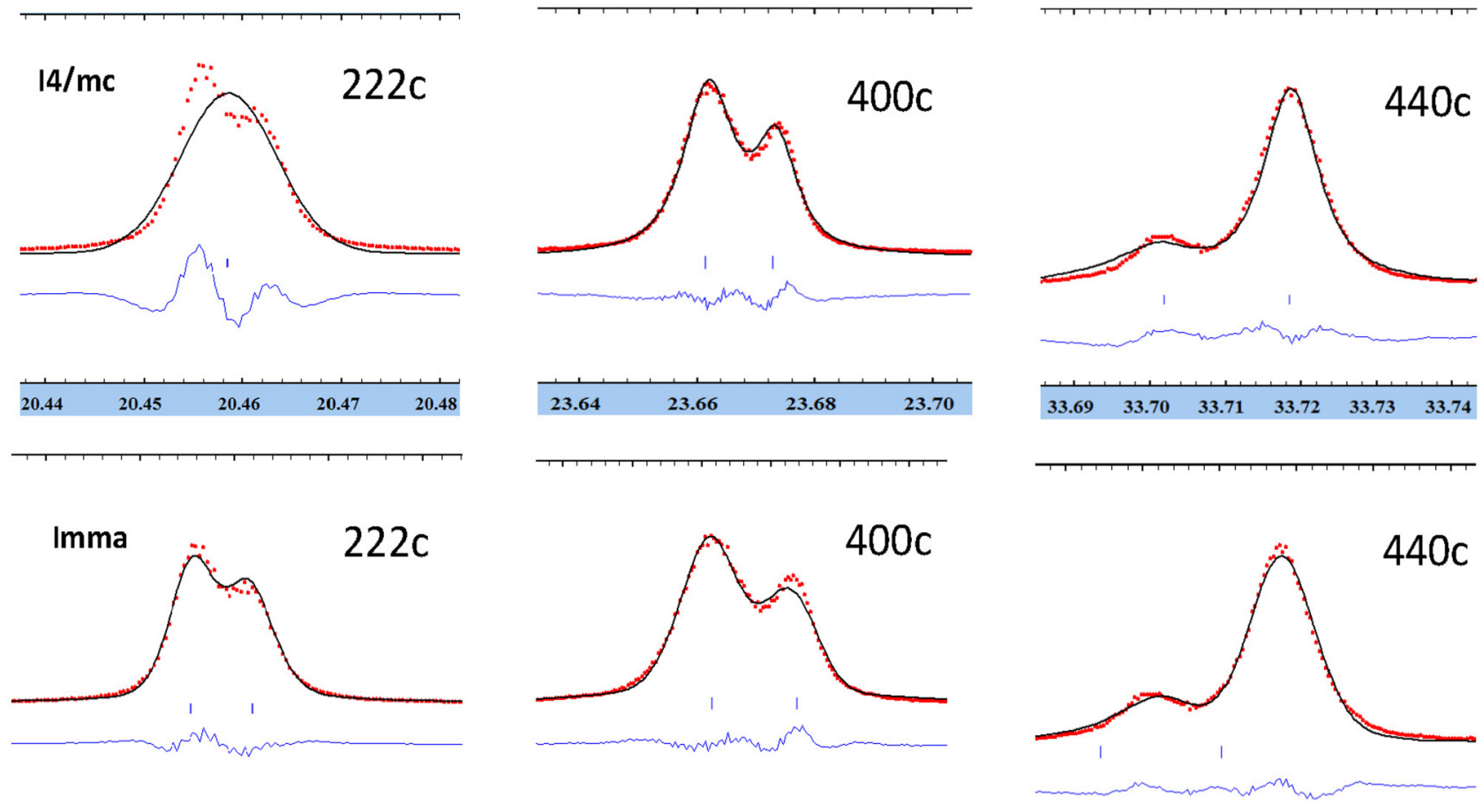

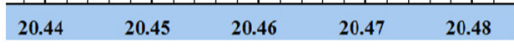

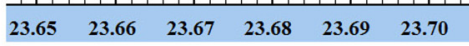

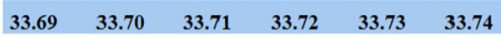

FIG. 3. The x-ray synchrotron selected Bragg peaks at $10 \mathrm{~K}$ of micrometric artificial CSS STO, with calculated lines and difference using the final structural Imma model (see below). It is clearly seen that 222c peaks cannot be explained by a tetragonal symmetry.

insert of Fig. 5) from split Bragg peaks and superstructure peaks gives a precise measurement of the critical temperature toward the untilted cubic phase.

On the contrary in natural CCS samples, all diffraction peaks could be indexed with the classical ferroelastic tetragonal phase. A careful analysis of the full patterns revealed this systematic twofold splitting of $H 00$ and $H H H$ and the need to use at low temperature an orthorhombic distortion (see Table I) in which the axis $a$ and $b$ are rotated from the cubic axis by an $45^{\circ}$, i.e., $a^{\prime} \approx a \sqrt{2}, b^{\prime} \approx a \sqrt{2}$ (i.e., three lattice parameters, whose temperature dependence is shown in Fig. 5, in a pseudocubic lattice) to index and correctly fit all peaks.

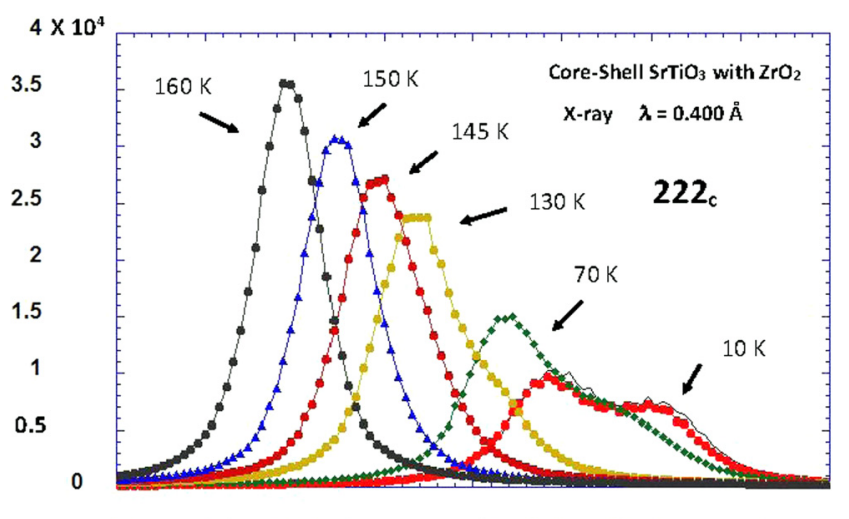

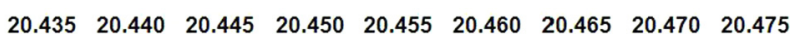

FIG. 4. Temperature evolution of the splitting of the 222c Bragg peak using x-ray synchrotron.
Also, the disappearance of this additional distortion is observed at the same temperature at which the $(H 00)$ distortion and the superstructure peaks disappear. This demonstrates in artificial CSS samples the existence of a ground state lower than the one in natural CSS STO or in bulk single crystal, with both tilting of oxygen octahedral and orthorhombic distortion. This orthorhombic ground state transforms into the cubic phase via a transition at temperatures significantly higher (up to $160 \mathrm{~K}$ in some cases) than the tetragonal-cubic temperature $(105 \mathrm{~K})$.

Stokes et al. [13] have applied group theoretical methods to compute all possible perovskite structures that can appear from the prototype cubic phase via ferroelectric cation displacements and/or tiltings of oxygen octahedra. This results in a list of 61 different structures with associated space groups, tilts systems, cation displacements, and irreducible representations. If no consideration of possible ferroelectric shifts should be done, we could ignore the corresponding space groups; however, as STO is "at the edge" of a possible ferroelectric phase, as explained in the Introduction, we preferred to consider this possibility. We have used this paper to analyze our synchrotron data, as well as neutron diffraction data collected from the same samples because it is a technique highly sensitive to oxygen displacements. Figure 6 shows a full pattern at $2 \mathrm{~K}$. Careful examination of the pattern reveals additional peaks (superstructure peaks, Fig. 7) compared to the room temperature situation. Moreover, only superstructure peaks corresponding to antiphase tilting were observed (Figs. 7 and 8), and this reduces the possibility to four different orthorhombic structures (three of them with 
TABLE I. Splitting of some simple $h k l$ Bragg peaks induced by different lowering of symmetry from the cubic phase, for some "classical" examples. In the $\mathrm{Cm}$ monoclinic and in the $\mathrm{Cmm} 2$ orthorhombic phases, the $c$ axis has the same direction as in the cubic phase, but the $a$ and $b$ axes are rotated by $45^{\circ}$ compared to the cubic axis, because, for instance, the $a$ monoclinic is the vector sum of $a$ and $b$ (pseudo) cubic axis (therefore its modulus is equal to $a \sqrt{2}$ ). Regarding the splitting, starting from the cubic phase, if we consider, for instance, the orthorhombic symmetry, the $H 00$ cubic peaks split into two families, i.e., with different distances, the $H H O$ cubic peaks split into three families, and the $H H H$ cubic peaks into two families. Consideration of this table allows us to discriminate among the most common cases of lowering of symmetry.

\begin{tabular}{|c|c|c|c|c|c|c|}
\hline \multirow{2}{*}{$\begin{array}{l}\text { Symmetry and } \\
\text { space Group }\end{array}$} & \multirow{2}{*}{$\begin{array}{l}\text { Cubic } \\
P m-\overline{3} m\end{array}$} & \multirow{2}{*}{$\begin{array}{l}\text { Rhombohedral } \\
\qquad R 3 m\end{array}$} & \multirow{2}{*}{$\begin{array}{l}\text { Tetragonal } \\
P 4 m m\end{array}$} & \multicolumn{2}{|c|}{ Monoclinic } & \multirow{2}{*}{$\begin{array}{l}\text { Orthorhombic } \\
\qquad m m 2\end{array}$} \\
\hline & & & & $P m$ & $\mathrm{Cm}$ & \\
\hline $\begin{array}{l}\text { Lattice parameters } \\
\text { and angles }\end{array}$ & $a$ & $a, \alpha$ & $a, c$ & $a, b, c, \beta$, & $a \sqrt{2}, b \sqrt{2}, c, \beta$ & $a \sqrt{2}, b \sqrt{2}, 2 c$ \\
\hline \multirow[t]{9}{*}{$h k l$ Miller indexes } & $H 00$ & $H 00$ & $H 00$ & $H 00$ & $00 H$ & $00 H$ \\
\hline & & & $00 H$ & $\begin{array}{l}0 H 0 \\
00 H\end{array}$ & $H H 0$ & $H H O$ \\
\hline & $H H O$ & $H H O$ & $H H O$ & $H H O$ & $\mathrm{HHH}$ & $2 H 00$ \\
\hline & & $\bar{H} H 0$ & $\mathrm{OHH}$ & $\mathrm{HOH}$ & $H H \bar{H}$ & $02 H 0$ \\
\hline & & & & $0 H \bar{H}$ & $02 H 0$ & $H H H$ \\
\hline & & & & $H 0 \bar{H}$ & $2 \mathrm{H} 00$ & \\
\hline & $H H H$ & $H H H$ & $H H H$ & $H H H$ & $2 \bar{H} 0 H$ & $2 H 0 H$ \\
\hline & & $H H \bar{H}$ & & $H H \bar{H}$ & $02 H H$ & $02 H H$ \\
\hline & & & & & $2 H 0 H$ & \\
\hline
\end{tabular}

same space groups; see Table II). Although we did not observe supplementary distortions, which should have indicated a ground state lower than orthorhombic, we have also tested all other possibilities, i.e., eight monoclinic and two triclinic structures using both synchrotron and neutron data: only the four different orthorhombic structures gave satisfactorily and equivalent agreement factors.

At this point, the new antiferrodistortive orthorhombic ground state is clearly demonstrated thanks to the high synchrotron resolution, the neutron diffraction, and the good quality of the fitting (see Figs. 2, 3, 6, 7, and 8), resulting in good agreement factors for both neutron and x-ray structural analysis.

The temperature dependence of splitting and associated width of the Bragg peaks (Fig. 5), superstructure peaks, and good quality of fittings (as well as Raman and hyper-Raman data not shown here) indicate a direct transition from cubic to orthorhombic phase. Although we could not completely exclude a temperature range below $5 \mathrm{~K}$ for another transformation, the modeling (see below) also supports this observation. At this stage, it is impossible to choose among the four possible orthorhombic models. This is, however, of crucial importance, as one of them is not a ferroelectric structure, whereas the three others are. Fortunately, we could go deeper in the analysis by using the cations shifts $\delta_{k}$ from the high symmetric cubic phase obtained both from x-ray and neutron diffraction (for higher precision of oxygen positions), to calculate the magnitude of polarization, via effective charges. Indeed, in the case of a $k$ ion with an apparent $Z^{\prime \prime}{ }_{k}$ charge and a $\delta_{k}$ displacement from cubic position, the local polarization is $Z^{\prime \prime}{ }_{k} \delta_{k}$ and the total polarization is

$$
P=\frac{e}{V} \sum_{k} Z^{\prime \prime}{ }_{k} \delta_{k}
$$

where $e$ is the electron charge and $V$ the lattice volume (obtained also from structural analysis). The apparent charges are different from the charge of a rigid ion due to local polarization. They have been calculated and experimentally verified by Hewat [14] in the case of many ferroelectric compounds: for STO, $Z^{\prime \prime}{ }_{k}$ is 3.6, 5.7, and -3.1 for $\mathrm{Sr}^{2+}, \mathrm{Ti}^{4+}$, and $\mathrm{O}^{2-}$ electron, respectively.

In all three cases, for all artificial CSS samples, we have titanium inside its oxygen octahedral and strontium in its oxygen cuboctahedra displacements below $0.04 \AA$ and a polarization below $4 \mu \mathrm{C} \mathrm{cm}^{-2}$, to be compared with the polarization calculated in the same way [15] in BT0 and in PTO, which are 35 and $75 \mu \mathrm{C} \mathrm{cm}^{-2}$, respectively. Moreover, as diffraction probes the whole sample, this value includes both the core and shell contribution.

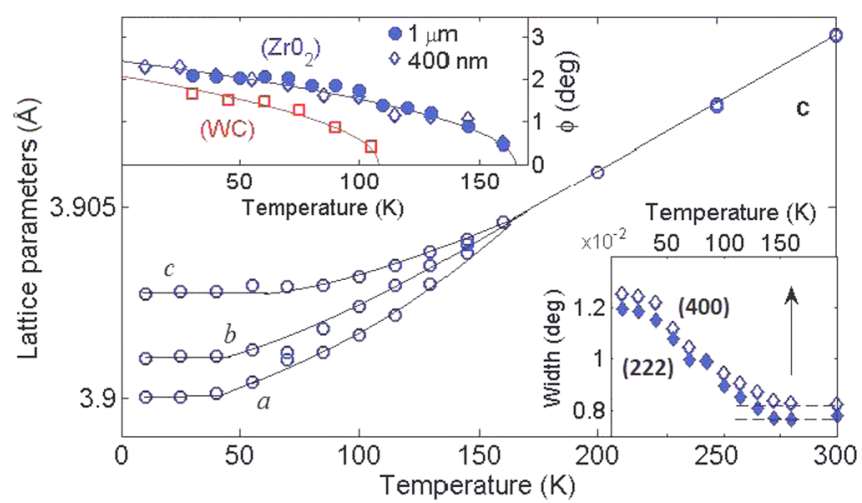

FIG. 5. Temperature-dependence of pseudocubic lattice parameters micrometric artificial CSS STO, plotted in pseudocubic cell, obtained from synchrotron diffraction experiment. Top left inset: tilting angle $\phi$ in function of temperature obtained by neutron diffraction in natural core-shell STO sample, with the classical

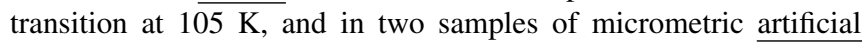
CSS STO (1 $\mu \mathrm{m}$ and $400 \mathrm{~nm})$ with the orthorhombic-cubic transition close to $160 \mathrm{~K}$. Bottom right inset: temperature-dependence of the full width at half maximum for both (222) and (400) Bragg peaks of micrometric artificial CSS STO indicating a probable direct transition of the orthorhombic phase at $\approx 160 \mathrm{~K}$ for these samples. 


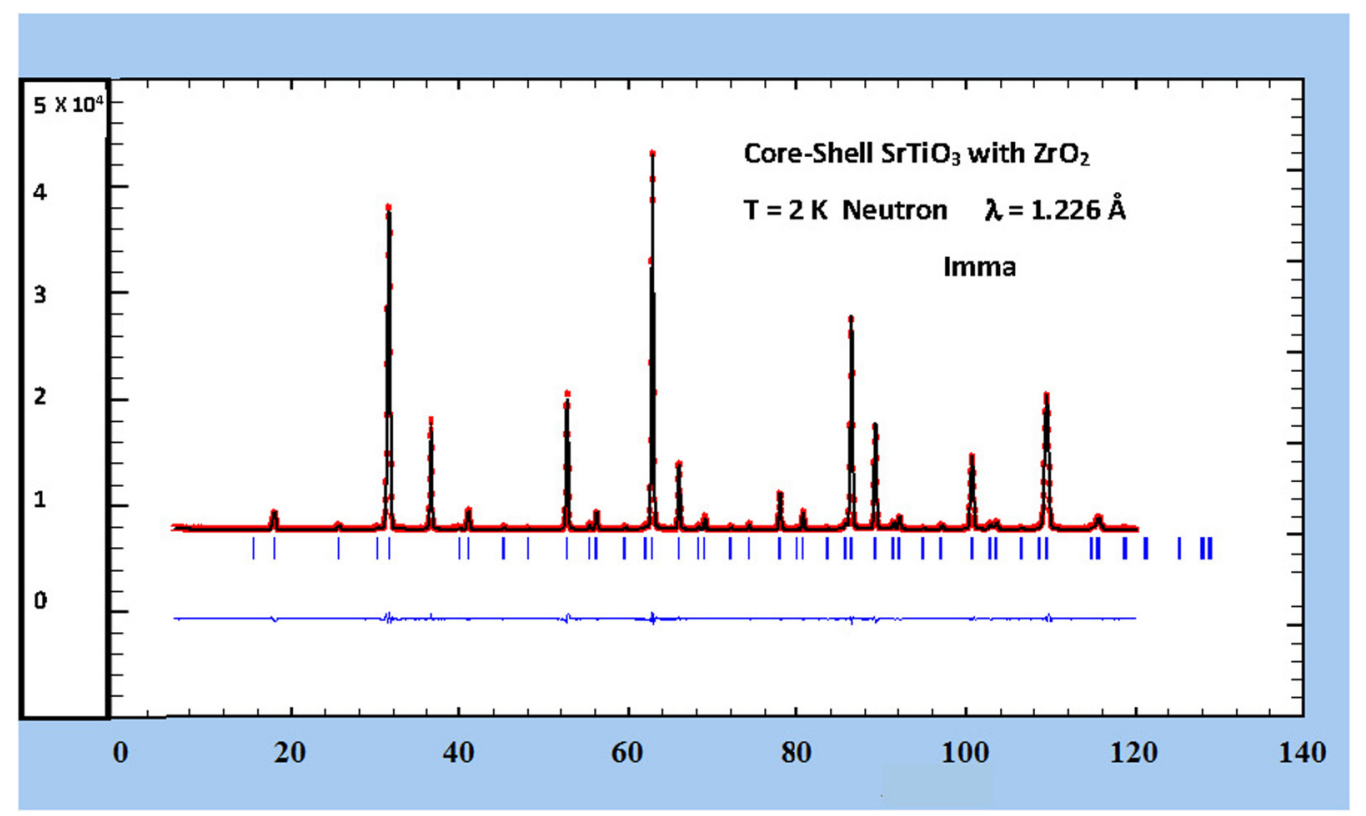

FIG. 6. Neutron full pattern at $10 \mathrm{~K}$ of micrometric artificial CSS STO, with calculated lines and difference using the final structural Imma model (see below).
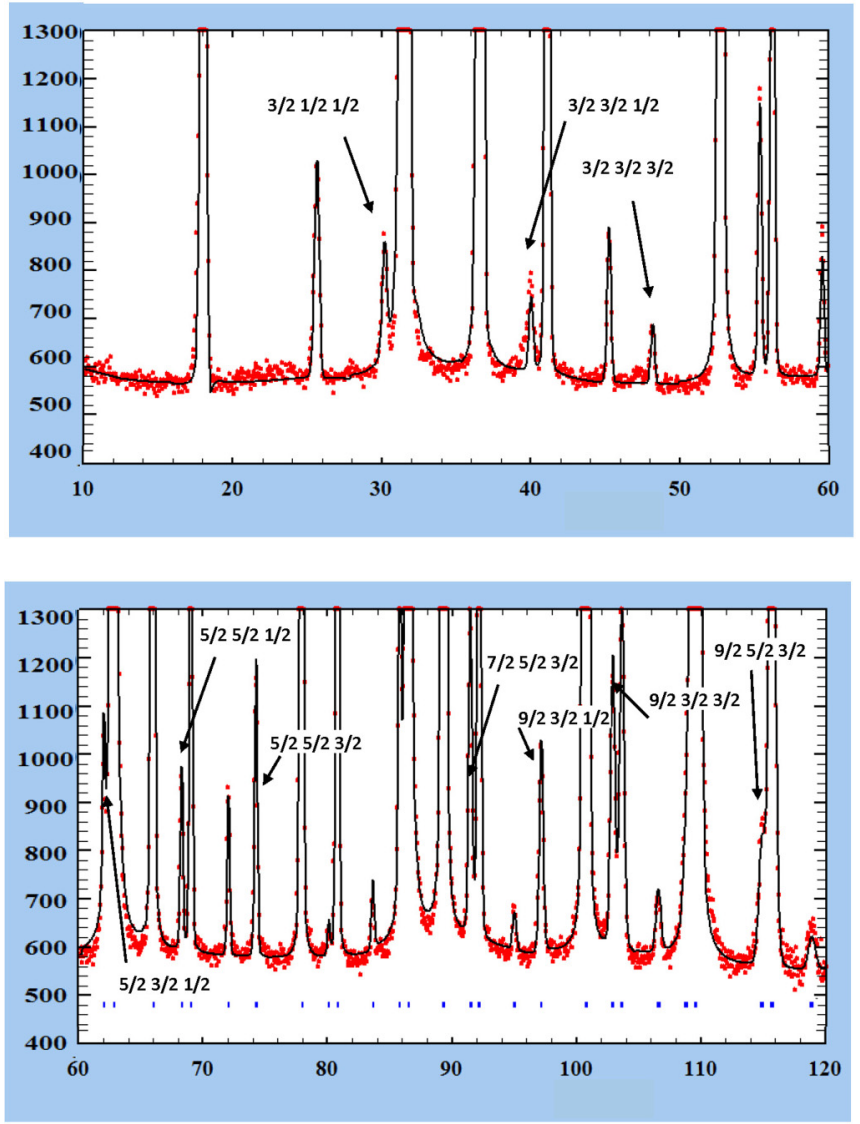

FIG. 7. Enlarged part of Fig. 6 showing all new (superstructure) peaks that appears during the transition in all types of samples, as well as the calculated line from the Imma model. The Miller indexes are given in the high temperature pseudocubic phase: They indicate that only antiphase rotations appears during the transition.
In the description of natural CCS by Petzelt [2], a (frozen) polarization appears at the shell and is responsible of the dielectric properties of the (unpolarized) core. Therefore, it can be concluded that the orthorhombic structure of CSS STO is either nonferroelectric or very weakly ferroelectric, with an associated polarization typically ten or 20 times less than classical ferroelectric compounds. The most probable space group is therefore Imma, which has two pseudocubic axes of out-of-phase tilting of octahedra, whereas tetragonal STO has only one such axis.

\section{Modeling with Landau theory}

We have now to turn to modeling to obtain insight on the physical origin of these observations. Modeling of core-shell ferroelectric nanoparticles and nanoceramics using a combination of electrodynamics and effective medium approximation (such as developed in Ref. [16]), as well as the Landau theory and ab initio-based calculation have enabled us $[6,17]$ to study dielectric properties and structural changes and to discuss the existence and role of vortex induced by natural and artificial CSS. It was predicted that changes induced by the existence of a shell can be explained on the basis of its sole dielectric properties. The STO, however, is not ferroelectric, and there is clearly a need of CSS to model the size dependence of permittivity, as demonstrated in Ref. [2]. This points out the existence of a core shell in all kinds of STO nanoparticles, whatever way they are synthesized. However, the phase transition that normally occurs at $105 \mathrm{~K}$ is purely ferroelastic and, eventual coupling with a weak polarization (if any), cannot explain the observed low symmetry phase in artificial CSS. Indeed, charged surfaces of STO grains due to oxygen vacancies create a nonhomogeneous radial polarization [2] (assuming spherical particles), which does not propagate an electric field inside the core. On the other hand, there have been contradicting reports on STO 
TABLE II. The four different orthorhombic phases that are the possible structural models for the new phase evidenced by synchrotron diffraction. The first line is the space group, and the second line shows the Glazer-Stokes notation and the numbers of the space group from Ref. [13] in a pseudocubic setting, where the upper index 0 or - indicates no tilt or antiphase tilt, respectively, and the lower index is the direction and sign of ferroelectric shifts. The third and fourth lines show the notation: the corresponding axis of tilt and the direction of eventual ferroelectric shifts. The fifth line is a typical agreement factor for the structural refinements, profile factor $R_{\mathrm{wp}}$ and goodness of fit (i.e., $\chi^{2}$ ) within the different hypothesis. The tetragonal phase is also shown as a reference.

\begin{tabular}{|c|c|c|c|c|c|}
\hline Space group & Imma & $\operatorname{Ima} 2$ & $\operatorname{Ima} 2$ & $\operatorname{Ima} 2$ & $I 4 / \mathrm{mcm}$ \\
\hline \multirow{2}{*}{$\begin{array}{l}\text { Glazer-Stokes notation and } \\
\text { reference numbers of the } \\
\text { space group from Ref. [13] }\end{array}$} & $a_{0}^{0} b_{0}^{-} b_{0}^{-}$ & $a_{+}^{0} a_{+}^{0} c_{0}^{-}$ & $a_{0}^{-} a_{0}^{-} c_{+}^{0}$ & $a_{+}^{-} a_{+}^{-} c_{0}^{0}$ & $a_{0}^{0} a_{0}^{0} c_{0}^{-}$ \\
\hline & 7 & 39 & 40 & 41 & 6 \\
\hline Tilting axis & $\langle 011\rangle$ & $\langle 001\rangle$ & $\langle 110\rangle$ & $\langle 110\rangle$ & $\langle 001\rangle$ \\
\hline Ferroelectric shifts $\delta$ & None & $\langle 100\rangle$ and $\langle 010\rangle$ & $\langle 001\rangle$ & $\langle 100\rangle$ and $\langle 010\rangle$ & None \\
\hline \multirow{2}{*}{$R_{\mathrm{wp}} \mathrm{X}$-ray goodness of fit } & 15.18 & 15.36 & 15.27 & 15.23 & 17.00 \\
\hline & 1.56 & 1.56 & 1.57 & 1.57 & 1.75 \\
\hline \multirow[t]{2}{*}{$R_{\mathrm{wp}}$ neutron goodness of fit } & 3.34 & 3.49 & 3.50 & 3.66 & 3.37 \\
\hline & 2.48 & 2.60 & 2.60 & 2.72 & 2.52 \\
\hline
\end{tabular}

under pressure of the possible existence of an orthorhombic phase, which were afterward disregarded by experiments and calculations [18]. In this paper, these authors used a sixth power Landau potential with a linear-quadratic coupling between the order parameter and the spontaneous strains and a pure elastic energy term. However, they restricted their study to a one-dimensional order parameter, as they did not observe experimentally any supplementary phase in addition to the $P m 3 m$ and $I 4 / \mathrm{mcm}$ ones. They also include a $P_{r}$ hydrostatic pressure term that is linear in the form $\lambda P_{r}(\lambda$ is elastic constant dependent) to account for the shifts of critical temperature with pressure.
We started from a potential used originally for STO thin films by Chen [19] et al. assuming no homogeneous polarization from our structural results, therefore neglecting all the polarization terms. It is possible to account for the lowering from tetragonal toward orthorhombic structure, as well as the strong shifts in the phase transition temperature by using a potential up to the fourth order as

$$
\begin{aligned}
F= & \beta_{1}\left(q_{x}^{2}+q_{y}^{2}+q_{z}^{2}\right)-K_{1}\left(n_{x} q_{x}^{2}+n_{y} q_{y}^{2}+n_{z} q_{z}^{2}\right) \\
& +\beta_{11}\left(q_{x}^{4}+q_{y}^{4}+q_{z}^{4}\right) \\
& +\left(\beta_{12}-K_{2}\right) \cdot\left(q_{x}^{2} q_{y}^{2}+q_{y}^{2} q_{z}^{2}+q_{x}^{2} q_{z}^{2}\right) .
\end{aligned}
$$

Core-Shell $\mathrm{SrTiO}_{3}$ with $\mathrm{ZrO}_{2} \quad$ Neutron $\quad \lambda=1.226 \AA \quad$ Imma

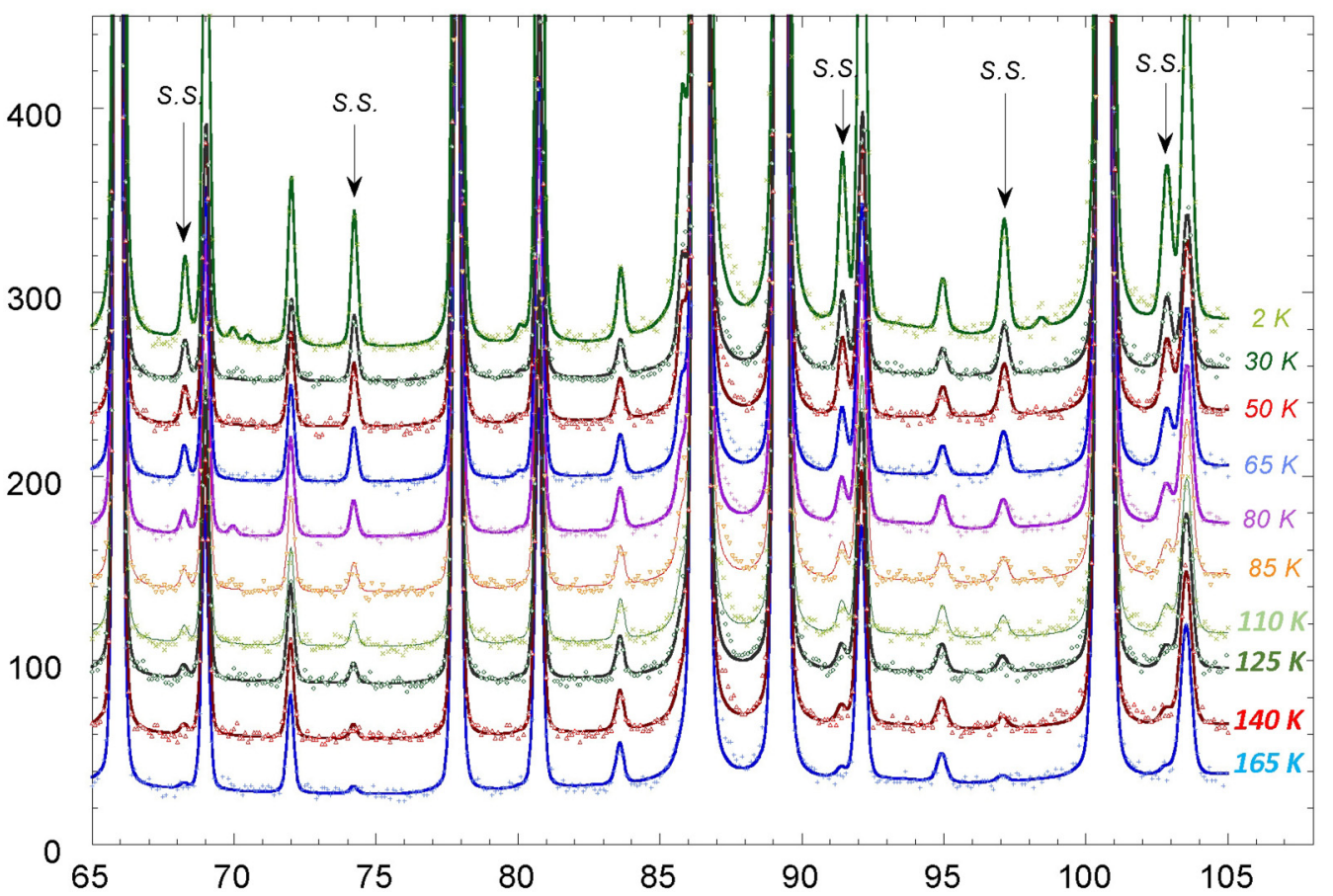

FIG. 8. Temperature evolution of enlarged part of Fig. 6 showing the evolution of superstructure peaks, as well as the calculated line from the Imma model up to the new (shifted) critical temperature. 
Compared to what is classically done in bulk STO, we have taken a three-dimensional AFD order parameter with $q_{i}$ components, and we have included two new terms, $K_{1}$ and $K_{2}$. The $K_{1}$ term is similar to the pressure term used in Ref. [18]. As Uchino et al. [20] showed that in small particles, the surface tension is analog to a hydrostatic pressure, $K_{1}$ accounts in the case of small particles for both surface tension and normal component of stress due to the shell and renormalizes the low order $\beta_{1}$ coefficient of the thermodynamical potential. The $n_{i}$ terms with $0 \leqslant n_{i} \leqslant 1$ allow taking into account the anisotropic (ellipsoid) shape of particles. The important point here is that using this $K_{1}$ term induces a shift of the ferroelastic transition toward high temperatures. However, no additional phase transition can emerge from this simple renormalization. Therefore, a second necessary ingredient is to include nonhydrostatic strain term $K_{2}$ corresponding to shear strain induced by the shell to obtain lower symmetry ground states.

A detailed analysis will be published elsewhere but here we give some main results. We have made calculations for temperature $K_{2}$ phase diagrams with a $K_{1}$ value of $2.10^{28} \mathrm{~m}^{-2}$, which is equivalent to $2 \mathrm{GPa}$ pressure and corresponds to a shift of the critical temperature to $140 \mathrm{~K}$ (experimentally observed). First, when particles of spherical shapes are considered, i.e., all $n_{i}=1$, the phase diagram is as follows [Fig. 9(a)]: for $K_{2} \leqslant 5.10^{49}$, the cubic $\left(q_{x}=q_{y}=q_{z}=\right.$ $0, P m \overline{3} \mathrm{~m})$-tetragonal $\left(I 4 / \mathrm{mcm}, q_{z} \neq 0, q_{x}=q_{y}=0\right)$ transition takes place below $140 \mathrm{~K}$ for all values of $K_{2}$, whereas for $K_{2} \geqslant 5.10^{49}$, a direct transition from cubic to rhombohedral $R \overline{3} c$ phase (with $q_{x}=q_{y}=q_{z}$ ) appears below $140 \mathrm{~K}$. This phase was not observed during the experiments.

However, as soon as slightly nonspherical particles are considered, there are complete changes. In Fig. 9(b), we plot the phase diagram for $n_{x}=n_{y}=1, n_{z}=0.95$, i.e., disc-shaped particles. Above $140 \mathrm{~K}$, the cubic phase is observed for all values of $K_{2}$. Below 140 $\mathrm{K}$, a transition to tetragonal phase takes place for $K_{2} \leqslant 5.10^{49}$, as in the case of natural CSS STO. However, for $5.10^{49} \leqslant K_{2} \leqslant 6.510^{49}$, a direct cubic-antiferrodistortive orthorhombic $\left(q_{x}=q_{y} \neq 0, q_{z}=0\right.$, i.e., Imma) transition is obtained, as we have observed in artificial CSS. Therefore, the orthorhombic phase is induced by the slightly nonisotropic surface tension and nonisotropic normal stress of the particles. In a similar manner, the report of an orthorhombic phase under pressure is most probably due to nonhydrostatic pressure during experiments, as suggested in Ref. [18]. Among the different new phases predicted in STO films [21], there is such an antiferrodistortive nonferroelectric orthorhombic phase.

In the very high shear strain region, i.e., for $K_{2}>6.5 .10^{49}$, a second transition to an antiferrodistortive phase $\left(q_{x} \neq 0 q_{y} \neq\right.$ $0 q_{z} \neq 0$, i.e., triclinic $\left.p \overline{1}\right)$ happens, which is not observed in our experiments. These high values of $K_{2}$ should be induced by highly "rough-shaped" particles, that is, highly non-ellipsoid-shaped particles in which high nonhydrostatic stress is retained. Temperature dependence of the $q_{i}$ order parameter has also been studied: In particular, whereas the already mentioned $K_{1}$ is responsible for the value of the critical temperature, competition between the $K_{1}$ and $K_{2}$ terms
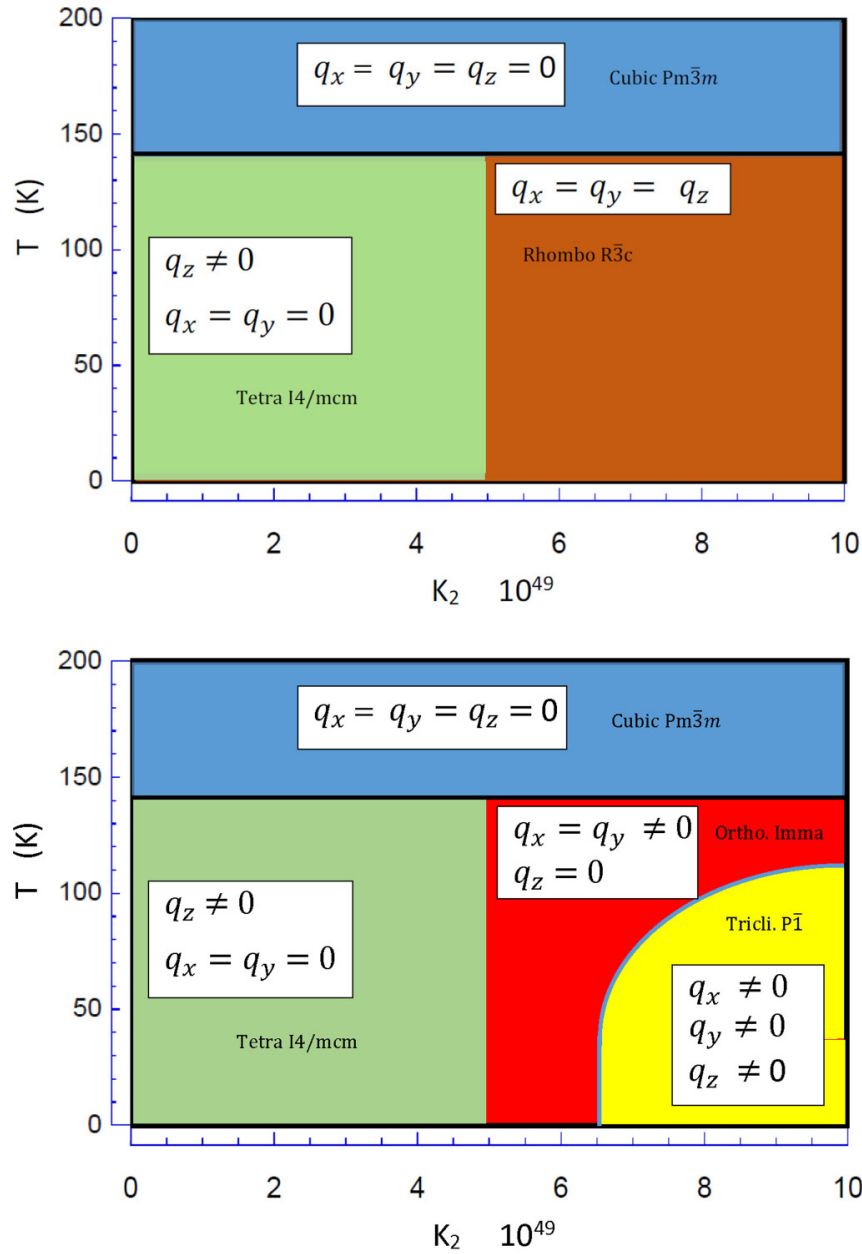

FIG. 9. Temperature- $K_{2}$ phase diagrams: Phase diagram calculated from Landau potential (see text), with a $K_{1}$ value of $2.10^{28} \mathrm{~m}^{-2}$, equivalent to a $2 \mathrm{GPa}$ pressure and corresponding to a shift of critical temperature to $140 \mathrm{~K}$. The $\mathrm{x}$-axis is the $K_{2}$ coefficient corresponding to shear strain induced by the shell, and $Y$ axis is the temperature. The pictures show the results (a) for spherical $\left(n_{i}=1\right)$ and (b) ellipsoid-shaped particles $\left(n_{x}=n_{y}=1, n_{z}=0.95\right)$. Depending on the value of $K_{2}$, below $140 \mathrm{~K}$ a tetragonal, orthorhombic, or triclinic phase is obtained.

determine the saturation value of the order parameter (i.e., the oxygen octahedral tilting angle $\phi)$. For some combinations of $K_{1}$ and $K_{2}, \phi$ values similar to bulk $\left(\approx 2.4^{\circ}\right)$, but with strong temperature shifts, can be obtained. This was observed in a previous neutron study [22] but with no explanation at that time. When calculations are performed with higher values of anisotropy (for instance $n_{x}=n_{y}=1, n_{z}=0.90$ or 0.85 ), the critical strain for the orthorhombic-triclinic phase is increasing much faster than the tetragonal-orthorhombic one, i.e., the existence range of the orthorhombic phase increases. Calculation with a nanorod type anisotropy, for instance $n_{z}=0.99, n_{x}=n_{y}=1$, also shows above a critical strain the appearance of the orthorhombic phase.

In conclusion, a new orthorhombic antiferrodistortive phase was induced in STO by creating an artificial CSS. Our results point out that the crucial part is played by the shear strain induced by coating the surface of STO and the anisotropic 
shape of particles for the lowering of ground state. Creating a specific CSS is therefore an easy way to tailor structural properties and to stabilize new phases that cannot exist in bulk material, just like film deposition on a substrate.

\section{ACKNOWLEDGMENTS}

We thank C. Giacobbe and all staff members of ID22 spectrometer for their help during the $\mathrm{x}$-ray diffraction experiment at European Synchrotron Radiation Facility, Grenoble, France.
[1] J. M. Kiat, C. Bogicevic, F. Karolak, G. Dezanneau, N. Guiblin, W. Ren, L. Bellaiche, and R. Haumont, Phys. Rev. B 81, 144122 (2010).

[2] J. Petzelt, Ferroelectrics 400, 117 (2010).

[3] J. Carreaud, C. Bogicevic, B. Dkhil, and J. M. Kiat, Appl. Phys. Lett. 92, 242902 (2008).

[4] S. L. Swartz, T. R. Shrout, W. Schulze, and L. E. Cross, J. Am. Ceram. Soc. 67, 311 (1984).

[5] A. V. Kimmel, J. Íñiguez, M. G. Cain, and V. P. Sushko, J. Phys. Chem. Lett. 4, 333 (2013).

[6] M. Anoufa, J. M. Kiat, I. Kornev, and C. Bogicevic, J. Appl. Phys. 113, 054104 (2013).

[7] M. Anoufa, J. M. Kiat, and C. Bogicevic, Appl. Phys. Lett. 107, 172902 (2015).

[8] S. H. Baker, M. Roy, S. C. Thornton, M. Qureshi, and C. J. Binns, J. Phys.: Condens. Matter 22, 385301 (2010).

[9] A. Yamanaka, K. Kataoka, Y. Inaba, K. Inoue, B. Hehlen, and E Courtens, Europhys. Lett. 50, 688 (2000).

[10] Y. Uesu, R. Nakai, J. M. Kiat, C. Ménoret, M. Itoh, and T. Kyomen, J. Phys. Soc. Jpn. 73, 1139 (2004).

[11] H. Yokota, Y. Uesu, C. Malibert, and J. M. Kiat, Phys. Rev. B 75, 184113 (2007).
[12] V. Petricek, M. Dusek, and L. Palatinus, Z. Kristallogr. 229, 345 (2014).

[13] H. T. Stokes, E. H. Kisi, D. M. Hatch, and C. J. Howard, Acta Crystallogr. B 58, 934 (2002).

[14] A. W. Hewat, J. Phys. C 6, 1074 (1973).

[15] C. Malibert, B. Dkhil, J. M. Kiat, D. Durand, J. F. Bérar, and A. Spasojevic-de Biré, J. Phys.: Condens. Matter 9, 7485 (1997).

[16] A. Y. Emelyanov, N. A. Pertsev, S. Hoffmann-Eifert, U. Bottger, and R. Waser, J. Electroceram 9, 5 (2002).

[17] M. Anoufa, J. M. Kiat, I. Kornev, and C. Bogicevic, Phys. Rev. B 88, 144106 (2013).

[18] M. Guennou, P. Bouvier, and J. Kreisel, Phys. Rev. B 81, 054115 (2010).

[19] L. Q. Chen, in Physics of Ferroelectrics: A Modern Perspective, edited by K. Rabe, Ch. H. Ahn, and J.-M. Triscone (SpringerVerlag, Berlin, Germany, 2007), pp. 363.

[20] K. Uchino, E. Sadanaga, and T. Hirose, J. Am. Ceram. Soc. 72, 1555 (1989).

[21] N. A. Pertsev, A. K. Tagantsev, and N. Setter, Phys. Rev. B 61, R825 (2000).

[22] J. M. Kiat, C. Bogicevic, P. Gemeiner, A. Al-Zein, F. Karolak, N. Guiblin, F. Porcher, B. Hehlen, L. Yedra, S. Estradé, F. Peiro, and R. Haumont, Phys. Rev. B 87, 024106 (2013). 